\title{
Incorporating Fluorine Substitution into Conjugated Polymers for Solar Cells: Three Different Means, Same Results
}

\author{
Mary Allison Kelly, ${ }^{\dagger, \#}$ Steffen Roland, ${ }^{\perp, \#}$ Qianqian Zhang, ${ }^{\dagger}$ Youngmin Lee, ${ }^{\ddagger}, \|$ Bernd Kabius, ${ }^{\S}$ Qing Wang, \\ Enrique D. Gomez, ${ }^{\ddagger}, \S$ Dieter Neher, ${ }^{*, \perp}$ and Wei You*,† \\ ${ }^{\dagger}$ Department of Chemistry, University of North Carolina at Chapel Hill, 125 South Road, Chapel Hill, North Carolina 27599-3290, \\ United States \\ ${ }^{\ddagger}$ Department of Chemical Engineering, ${ }^{\S}$ Materials Research Institute, and "Department of Materials Science and Engineering, The \\ Pennsylvania State University, 106 Fenske Lab, University Park, Pennsylvania 16802, United States \\ ${ }^{\perp}$ Institute of Physics and Astronomy, University of Potsdam, Karl-Liebknecht-Str. 24-25, 14476 Potsdam, Germany
}

ABSTRACT: Fluorinating conjugated polymers is a proven strategy for creating high performance materials in polymer solar cells, yet few studies have investigated the importance of the fluorination method. We compare the performance of three fluorinated systems: a poly(benzodithieno-dithienyltriazole) (PBnDT-XTAZ) random copolymer where $50 \%$ of the acceptor units are difluorinated, PBnDT-mFTAZ where every acceptor unit is monofluorinated, and a 1:1 physical blend of the difluorinated and nonfluorinated polymer. All systems have the same degree of fluorination (50\%) yet via different methods (chemically vs physically, random vs regular). We show that these three systems have equivalent photovoltaic behavior: $\sim 5.2 \%$ efficiency with a shortcircuit current $\left(J_{\mathrm{sc}}\right)$ at $\sim 11 \mathrm{~mA} \mathrm{~cm}{ }^{-2}$, an open-circuit voltage $\left(V_{\mathrm{oc}}\right)$ at $0.77 \mathrm{~V}$, and a fill factor (FF) of $\sim 60 \%$. Further investigation of these three systems demonstrates that the charge generation, charge extraction, and charge transfer state are essentially identical for

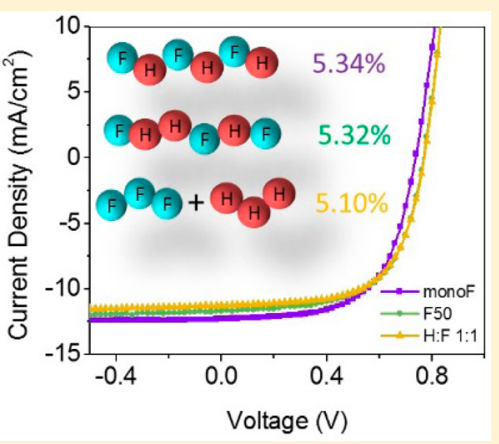
the three studied systems. Transmission electron microscopy shows no significant differences in the morphologies. All these data illustrate that it is possible to improve performance not only via regular or random fluorination but also by physical addition via a ternary blend. Thus, our results demonstrate the versatility of incorporating fluorine in the active layer of polymer solar cells to enhance device performance.

\section{INTRODUCTION}

Adding electronegative fluorine substituents to the backbone of the donor-conjugated polymer has become a popular and effective strategy for improving the power conversion efficiency (PCE) of polymer solar cells. ${ }^{1-10}$ Indeed, fluorination of the donor polymers can enhance all three key device output characteristics: open-circuit voltage $\left(V_{\text {oc }}\right)$, short-circuit current $\left(J_{s c}\right)$, and fill factor $(\mathrm{FF})$, via a variety of mechanisms. Typically, electronegative fluorine substituents can deepen the highest occupied molecular orbital (HOMO) level of the donor polymer, leading to a higher $V_{\text {oc }}$ in bulk heterojunction (BHJ) devices. ${ }^{11-14}$ Interestingly, this lowered HOMO level is often accompanied by a similar shift in the lowest unoccupied molecular orbital (LUMO) level, thereby maintaining the effective band gap of the donor polymer. ${ }^{1,8,13,15,16}$ In this case, if the dominant charge loss mechanisms can be mitigated by fluorination, the related $\mathrm{BHJ}$ devices would also demonstrate improved $J_{\text {sc }}$ and/or FF. ${ }^{11,17}$

In our earlier study on a series of polymers (PBnDT-DTBT) that have 0,1 , and 2 fluorine substituents on the benzothiadiazole (BT) unit, we showed that increased fluorination led to increased $V_{\mathrm{oc}} J_{\mathrm{sc}}$ and FF. ${ }^{11}$ While the improved $V_{\text {oc }}$ could be ascribed to the lower HOMO level, the
$J_{\text {sc }}$ and FF improved largely due to reduced recombination, which was traced to an increase in the change in dipole moment as well as improved morphology. Indeed, adding fluorine to conjugated polymers often has beneficial structural and morphological effects, including a more planar conjugated backbone and improved $\pi-\pi$ stacking, both of which can help improve charge transport. ${ }^{13,17-21}$ Furthermore, fluorination of the conjugated polymer can improve the face-on orientation of such polymers relative to the substrate and the domain purity in $\mathrm{BHJ}$ blends of the conjugated polymer and phenyl- $\mathrm{C}_{61}$-butyric acid methyl ester (PCBM), likely by excluding PCBM due to low miscibility. ${ }^{11,22}$ All these contribute to the higher power conversion efficiency (PCE) of the fluorinated polymer in $\mathrm{BHJ}$ devices when compared to a nonfluorinated counterpart. ${ }^{1,11,17}$ In fact, the current record-high efficiency was achieved with a fluorinated conjugated donor polymer, ${ }^{23,24}$ highlighting the impact and effectiveness of fluorination.

To further understand the performance-enhancing effect of fluorination, we recently synthesized a series of copolymers

Received: November 4, 2016

Revised: January 4, 2017

Published: January 4, 2017 
a)

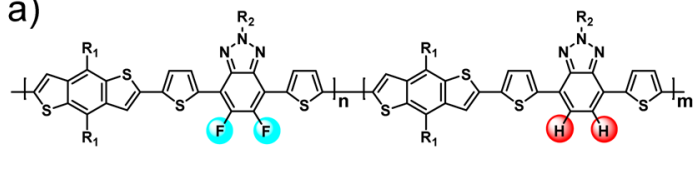

$\mathrm{F} 50(\mathrm{~m}: \mathrm{n}=1: 1)$

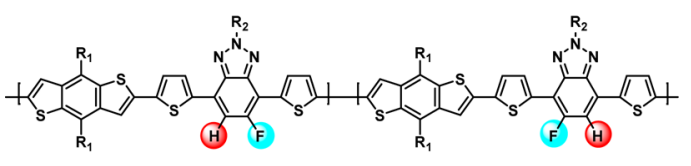

monoFTAZ

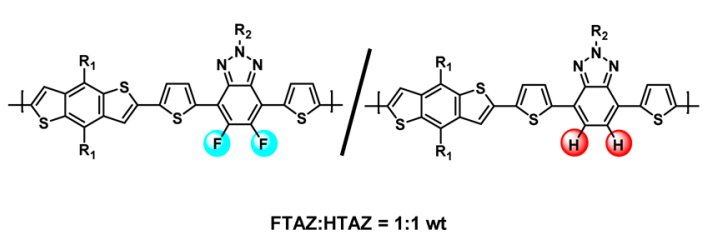

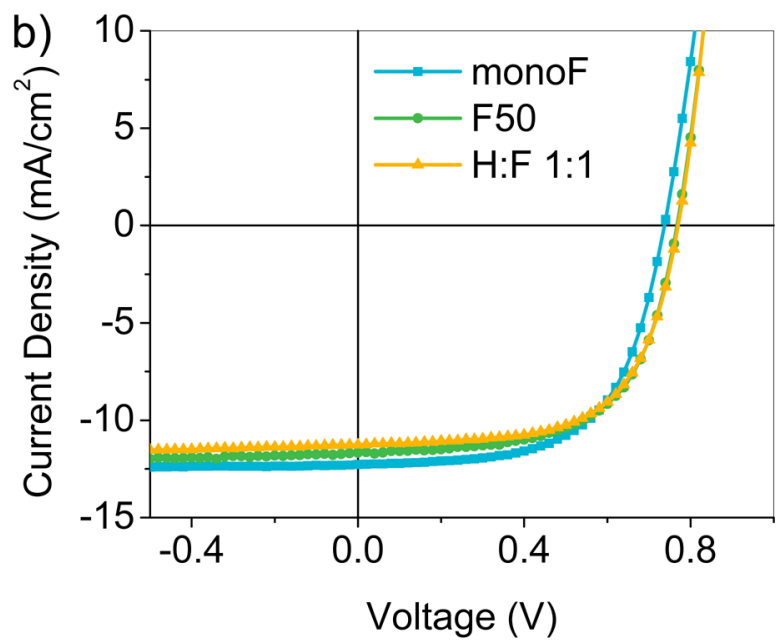

Figure 1. (a) Three different systems with $50 \% \mathrm{~F}$ content in the active layer. F50 is the random copolymer with $m: n$ being 1:1; due to the asymmetry of the monomer, monoFTAZ, the regular polymer, yet has two different orientations along the backbone; FTAZ:HTAZ is a physical blend that has two polymers at 1:1 weight ratio. (b) Representative $J-V$ curves for the $50 \%$ F blend solar cells.

based on poly(benzodithiophene-dithienyldifluorobenzotriazole) (PBnDT-FTAZ), ${ }^{1}$ where fluorine was incorporated randomly along the conjugated backbone to yield polymers with a systematically increased degree of fluorination. ${ }^{17} \mathrm{~A}$ careful and comprehensive investigation disclosed that domain size and purity, recombination kinetics, and charge generation all remained similar in the studied $\mathrm{BHJ}$ blends for all levels of fluorination, allowing us to directly link the fluorine impact to a single cause: the increased hole mobility due to improved $\pi-\pi$ stacking. ${ }^{17}$ Specifically, the increased hole mobility would lead to a faster charge extraction and thereby more attenuated bimolecular recombination, resulting in an increased FF (and therefore an improved PCE). Such improved hole mobility in the out of plane direction by the fluorination has also been observed in other cases. ${ }^{25}$

However, such a random copolymer has its own limitations. For example, in the F50 polymer (where $50 \%$ of the polymerized acceptor monomers were FTAZ, the fluorinated monomer), there could be "oligomeric" segments which structurally mimic PBnDT-FTAZ and segments mimicking PBnDT-HTAZ. This irregularity, intrinsic to the random copolymerization, could have a strong impact on the morphology and overall performance of the blend, as we observed in another study that compared a regular terpolymer and a random copolymer. ${ }^{26}$ Thus, in order to address the irregularity of the F50 polymer and further probe the effect that the method of fluorination has on the device performance, we decided to synthesize a polymer that also incorporated $50 \%$ fluorine along the backbone, poly(benzodithiophene-dithienylmonofluorobenzotriazole) (monoFTAZ), by fluorinating the TAZ unit with a single fluorine. This monoFTAZ polymer would ensure that the distribution of fluorine along the backbone is regular, with identical fluorine content (50\%) to that of the F50 polymer. In addition, similar to our previous study, ${ }^{26}$ a third scenario for introducing $50 \% \mathrm{~F}$ content into the active layer was also included in this study, namely a 1:1 (by weight) physical blend of PBnDT-HTAZ and PBnDT-FTAZ. This third scenario chemically mimics the F50 polymer and the monoFTAZ polymer but is a physical mixture rather than a covalently linked system. Therefore, these three scenarios construct an interesting library of polymeric systems that have identical F content (i.e., 50\% F) incorporated via different means (Figure 1a), allowing us to study the impact that the method of introducing fluorine to the system has on the device performance (i.e., random vs regular vs ternary).

Our study was also motivated by recent work on physical ternary blends, ${ }^{27-31}$ where a donor is mixed with two acceptors of different LUMO energy (or an acceptor is blended with two donors). While it was expected that the open-circuit voltage of such a ternary blend is limited by the donor-acceptor couple with the smaller HOMO-LUMO gap, some ternary blends had their $V_{\text {oc }}$ between those of the lower and of the higher bandgap binaries, with a distinct, more or less linear, dependence of $V_{\mathrm{oc}}$ on blend composition. Several mechanisms were proposed to explain this behavior, such as wave function delocalization over several molecules in an alloy, ${ }^{32}$ filling of a joint density of states, $^{33}$ long-range electrostatic effects, and a shift of the energy levels of the individual components caused by composition-dependent molecular aggregation. ${ }^{34}$ As a result, even if the two polymers in the ternary blend have no direct electronic interaction or measurable miscibility, there can be an indirect and yet large effect on Voc through the morphology. ${ }^{31}$ Here, the fluorinated TAZ units are introduced in three distinctly different fashions: regularly (monoFTAZ) or randomly (F50) along the same partially fluorinated backbone or through a 1:1 physical blend of fully fluorinated and nonfluorinated polymer chains ( $\mathrm{H}: \mathrm{F}$ 1:1). Thus, these three scenarios offer an ideal testbed to study the influence of the local distribution of the fluorinated units on the energetics of donor-acceptor blends and the resulting $V_{\mathrm{oc}}$.

Our results show that the monoFTAZ polymer (abbreviated as monoF) and the F50 polymer based solar cells give almost identical device characteristics in every respect, suggesting that any irregularities on the F50 backbone do not in fact reduce the performance. More interestingly, the photovoltaic device performance of the 1:1 blend of HTAZ:FTAZ is on par with those of the regular polymer and random polymer based devices, despite their physical differences.

Indeed, a detailed study of charge carrier generation, recombination, and extraction reveals identical properties for these three systems. Furthermore, the mobility improvement by chemically introducing fluorine substituents (i.e., random and 
regular) - as one would expect-is similarly achieved by physically blending the high mobility polymer (FTAZ) with the low mobility polymer (HTAZ). The combination of these effects indicates that the device performance is unaffected by the way the fluorinated sites are spatially distributed on a local scale. Thus, it may be possible to improve the mobility of other ternary blend systems (typically designed to improve light absorption) by ensuring that one of the components is a high mobility polymer (such as FTAZ).

\section{EXPERIMENTAL SECTION}

Photovoltaic Device Fabrication. All devices were fabricated on indium tin oxide that was cleaned via sonication in deionized water, acetone, and isopropyl alcohol and then subjected to a UV-Ozone treatment for $15 \mathrm{~min}$. A $\sim 50 \mathrm{~nm}$ layer of poly(3,4-ethylenedioxythiophene)-poly(styrenesulfonate) (PEDOT:PSS; PH500 from Clevios, used as delivered) was filter through a 0.45 poly(vinylidene fluoride) filter and spuncast at $2000 \mathrm{rpm}$ and annealed at $130{ }^{\circ} \mathrm{C}$ for 15 min. From this point all fabrication and characterization were carried out in a glovebox under nitrogen atmosphere. A solution of polymer(s): $\mathrm{PC}_{61} \mathrm{BM} \quad 1: 2$ ratio by wt, at a concentration of $12 \mathrm{mg} / \mathrm{mL}$ of polymer, in trichlorobenzene was stirred at $130{ }^{\circ} \mathrm{C}$ for $6 \mathrm{~h}$. $\mathrm{PC}_{61} \mathrm{BM}$ was purchased from Nano-C and used as received. The active layer was then spuncast through a 1 or $5 \mu \mathrm{m}$ poly(tetrafluoroethylene) filter at $400-800 \mathrm{rpm}$, to achieve a thickness of $200-250 \mathrm{~nm}$. The film was immediately dried under vacuum $(\sim 5 \mathrm{~mm} \mathrm{Hg})$ for $30 \mathrm{~min}$. The dried film was then loaded into the evaporator, where 30 $\mathrm{nm}$ of calcium was deposited via thermal evaporation, followed by $70 \mathrm{~nm}$ of aluminum, both at $3 \times 10^{-6}$ mbar. All photovoltaic devices were measured under AM 1.5G irradiation $(100 \mathrm{~mW} /$ $\mathrm{cm}^{2}$, Oriel 91160, $300 \mathrm{~W}$ ) calibrated using an NREL-certified standard silicon cell and recorded using a Keithley 2400 digital source meter. Reported values are the average of at least six devices with standard deviation. The thickness of the active layer was measured by an Alpha Step D-100 KLA -Tencor profilometer.

UV-vis absorbance spectra were taken on a Shimadzu UV2600 spectrophotometer of polymer films cast on glass from a $12 \mathrm{mg} / \mathrm{mL}$ solution in TCB.

Space Charge Limited Current (SCLC) Device Fabrication. Devices with the structure ITO/PEDOT:PSS/Active Layer $/ \mathrm{MoO}_{3}(10 \mathrm{~nm}) / \mathrm{Al}(70 \mathrm{~nm})$ were fabricated for measuring hole mobilities. Devices with the structure ITO/ ethoxylated-polyethylenimine (PEIE)/active layer/Ca (30 $\mathrm{nm}) / \mathrm{Al}(70 \mathrm{~nm})$ were fabricated for measuring electron mobilities. The active layer was cast and dried under the same conditions as was used for photovoltaic devices. For each set of devices, current was measured as a function of voltage in the dark from -1 to $5 \mathrm{~V}$ using a Keithley 2400 digital source meter. Mobilities were calculated using the Mott-Gurney Law as stated below

$$
J=\frac{9}{8} \varepsilon_{\mathrm{r}} \varepsilon_{0} \frac{V^{2}}{d^{3}} \mu
$$

where $\varepsilon_{\mathrm{r}}$ is the dielectric constant of the material (estimated to be about 3); $\varepsilon_{0}$ is the permittivity of free space; and $d$ is the thickness of the film The mobility was found by fitting the $J^{0.5}$ vs $V$ curve in the SCLC regime, taking into account the voltage drop and built-in potential from the ITO, PEDOT:PSS/PEIE, and metal electrodes.
Transmission Electron Microscopy Measurement. The thicker films for transmission electron microscopy (TEM) were cast under the same conditions used for the photovoltaic devices. Thinner films were cast by decreasing the concentration to $6 \mathrm{mg} / \mathrm{mL}$ of the polymer and spuncoat at 700-900 $\mathrm{rpm}$. The films were fabricated on Si wafers coated with $50 \mathrm{~nm}$ of unannealed PEDOT:PSS.

TEM measurements were performed on an FEI Titan G2 at the Materials Characterization Lab of the Pennsylvania State University. Bright-field images, thickness maps, sulfur maps, and carbon maps were acquired. The standard three-window method was used to obtain elemental maps. ${ }^{35}$

TDCF and BACE Measurements. Time-delayed collection field (TDCF) and bias-assisted charge extraction (BACE) were measured on devices fabricated under the same conditions used for the photovoltaic devices.

TDCF. Pulsed excitation from a diode pumped, Q-switched Nd:YAG laser (NT242, EKSPLA) with 6 ns pulse duration and $500 \mathrm{~Hz}$ repetition rate was used to generate charges in the device. A pulse generator (Agilent 81150A) was used to apply the pre- and collection bias in combination with a home-built amplifier. The current through the samples was measured via a $50 \Omega$ resistor and recorded with an oscilloscope (Yokogawa DL9140). The pulse generator was triggered with a fast photodiode (EOT, ET-2030TTL).

$B A C E .^{36}$ The same setup as for TDCF is used, except for the illumination conditions. Here the devices were illuminated with a high power $1 \mathrm{~W}, 445 \mathrm{~nm}$ laser diode (Insaneware) with a switch of time of about $10 \mathrm{~ns}$. The LED was operated at $500 \mathrm{~Hz}$ with a duty cycle of $90 \%$ of one period. This realized $1.8 \mathrm{~ms}$ of illumination before the diode was switched off for $200 \mu \mathrm{s}$. By this measurement, routine steady state conditions were established. After switching the light off, the voltage at the sample was reversed, and all charges were extracted. The extraction voltage was set to $-3 \mathrm{~V}$ to ensure extraction of all charges in the device.

\section{RESULTS AND DISCUSSION}

3.1. Electrochemical and Optical Properties. Figure 1a lists the three polymeric systems in this study. While PBnDTFTAZ (FTAZ), PBnDT-HTAZ (HTAZ), and $\mathrm{P}\left(\mathrm{BnDT}_{50^{-}}\right.$ $\mathrm{HTAZ}_{25}-\mathrm{FTAZ}_{25}$ ) (F50) were synthesized as previously reported, ${ }^{1,17}$ monoF was newly prepared (synthetic procedure and supporting analysis in Supporting Information: Figures S1-S7, Table S1). All polymers in this study had sufficiently high molecular weight (over $40 \mathrm{~kg} / \mathrm{mol}$, Table S2 in Supporting Information) to minimize any possible complication to the device performance. ${ }^{36}$ The optical band gap of monoF was 1.94 $\mathrm{eV}$, calculated from the UV-vis spectrum of a neat polymer film cast from trichlorobenzene (TCB) (Figure S9). This value is comparable to the band gaps of all other polymers in this study. ${ }^{1,17}$ The similar optical band gaps of all polymers, coupled with the similar absorbance for the polymer: $\mathrm{PC}_{61} \mathrm{BM}$ blends of all three 50\% F films (monoF, F50, and H:F 1:1), indicate that the difference in the method of introducing fluorine does not significantly affect the absorption properties of the blends (Figure S10). Therefore, any differences in performance are unlikely to be related to the light absorption.

Cyclic voltammetry (CV) was conveniently used to estimate the energy level of the highest occupied molecular orbital (HOMO) of the polymers of interest (Figure S8). We want to emphasize the estimative nature in using $\mathrm{CV}$ to access the HOMO level of conjugated polymers, as the numerical value of 
the frontier orbital levels can be influenced by the measurement itself and the calculation method used. ${ }^{37}$ In this study, the absolute values of the HOMO levels of FTAZ and HTAZ are both slightly higher than previously reported but still differ by $\sim 0.05 \mathrm{eV}$, consistent with our previous report (Figure 2). ${ }^{17}$ Interestingly, both F50 and monoF show HOMO levels between that of HTAZ and FTAZ, given the strong influence the fluorination has on the HOMO level (Table S2).

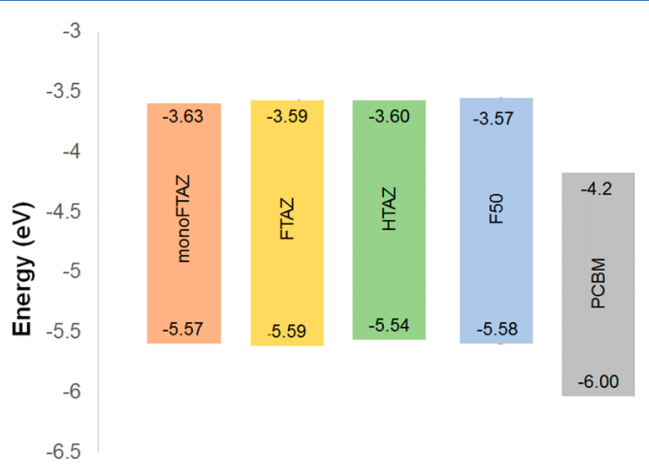

Figure 2. Energy levels of relevant polymers and PCBM.

3.2. Device Performance. To study the photovoltaic performance of these polymers, devices were fabricated with the conventional structure ITO/PEDOT:PSS / polymer(s): $\mathrm{PC}_{61} \mathrm{BM}(1: 2) / \mathrm{Ca} / \mathrm{Al}$ and measured under 1 sun condition with a $1.5 \mathrm{AM}$ solar simulator. The active layer was cast under the same condition for all devices. For all devices, thickness was maintained at 200-250 nm for accurate comparison. Results are tabulated in Table 1, with representative $J-V$ curves shown in Figure $1 \mathrm{~b}$. To our surprise, the performance for all three $50 \% \mathrm{~F}$ systems is remarkably similar. Specifically, the $J_{\text {sc }}$ of the monoF based device and the H:F 1:1 based device are very similar at $\sim 11 \mathrm{~mA} \mathrm{~cm}^{-2}$ (the $J_{\mathrm{sc}}$ of the F50-based cells is slightly higher at $11.6 \mathrm{~mA} \mathrm{~cm}^{-2}$, though the standard deviations suggest this is not a significant difference). The $V_{\mathrm{oc}}$ values of all three $50 \% \mathrm{~F}$ based devices are almost identical at $\sim 0.77 \mathrm{~V}$, and more interestingly, the fill factors of all three blends are between that of HTAZ and FTAZ at 60\%, which was expected for at least the F50 system. ${ }^{17}$ The external quantum efficiency (EQE) was also measured for these systems (Figure S11): all blends exhibit an EQE between 50 and 60\%. All these data suggest that regardless of the means of fluorination (random or regular, physical or chemical) a similar increase in performance over the nonfluorinated system is observed. While the similar device performance of monoF based cells and the F50 based ones is somewhat expected, given the equivalent fluorination in these two binary blend based $\mathrm{BHJ}$ cells, the strikingly similar device characteristics of $\mathrm{H}: \mathrm{F}$ 1:1 ternary blend based cells to those of monoF (and F50) are rather surprising, which prompted us to conduct further investigations.
The rather high fill factors and EQE suggest charge generation to be efficient and independent of field. To confirm this, the external generation efficiency (EGE) was measured as a function of bias, using time-delayed collection field (TDCF) experiments. Details about the setup and the experimental conditions can be found in previous studies and the Experimental Section. ${ }^{38-42}$ TDCF experiments reveal that the EGE is in a similar range $(70-80 \%)$ for all three $50 \% \mathrm{~F}$ blends at charge carrier densities comparable to one sun conditions and that the EGE is independent of the applied voltage (Figure S12), consistent with previous measurements on HTAZ, FTAZ, and its statistical copolymers in combination with $\mathrm{PC}_{61} \mathrm{BM}^{17}$ Though the exact mechanism of free charge generation at the donor-acceptor heterojunction is still unknown, ${ }^{43}$ recent work revealed correlations of EGE with morphological features, e.g., domain sizes and purities, ${ }^{4-46}$ hole delocalization on the polymer backbone, ${ }^{47}$ or the mobility of the slower carrier. ${ }^{48}$ Given the fact that all three systems studied are highly similar with respect to the absolute value and field dependence of EGE, none of these properties seem to depend on the way backbone fluorination is introduced into the blend.

3.3. Open-Circuit Voltage and CT Energy. As noted above, all three $50 \% \mathrm{~F}$ systems display very similar $V_{\text {oc }}$. It is now well established that the open-circuit voltage of organic $\mathrm{BHJ}$ solar cells is determined by the energy $E_{\mathrm{CT}}$ of the charge transfer state (CT state) and by the radiative and nonradiative losses. As shown by Vandewal et al., all three quantities can be determined from the external quantum efficiency spectra, $\mathrm{EQE}_{\mathrm{PV}}$ and $\mathrm{EQE}_{\mathrm{EL}}$ ( $\mathrm{EQE}$ for photovoltaics, and electroluminescence, respectively). ${ }^{49}$

The measured spectra, as plotted in Figure 3, are very similar for all three systems, indicating that the conditions determining

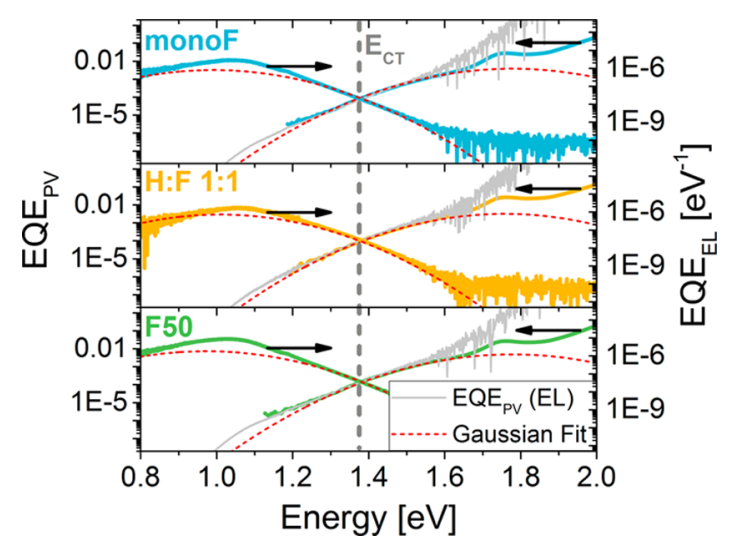

Figure 3. External quantum efficiency of the photocurrent and the electroluminescence for all three systems. Gaussian fits to the spectra are shown as red dashed lines, and the external quantum efficiency of the photocurrent calculated from the emission spectrum is shown as gray lines.

Table 1. PV Performance for the H:F Physical Blends as Well as the Four Polymers of Interest

\begin{tabular}{|c|c|c|c|c|c|}
\hline & thickness (nm) & $J_{\mathrm{sc}}\left(\mathrm{mA} / \mathrm{cm}^{2}\right)$ & $V_{\mathrm{oc}}(\mathrm{V})$ & FF (\%) & PCE (\%) \\
\hline HTAZ & $247 \pm 31$ & $10.4 \pm 0.4$ & $0.761 \pm 0.002$ & $49.7 \pm 0.7$ & $3.93 \pm 0.18$ \\
\hline FTAZ & $233 \pm 11$ & $11.5 \pm 0.4$ & $0.810 \pm 0.010$ & $72.5 \pm 1.1$ & $6.73 \pm 0.31$ \\
\hline monoF & $208 \pm 3$ & $11.0 \pm 0.5$ & $0.769 \pm 0.004$ & $62.9 \pm 1.4$ & $5.34 \pm 0.26$ \\
\hline $\mathrm{H}: \mathrm{F} 1: 1$ & $245 \pm 33$ & $10.8 \pm 0.4$ & $0.768 \pm 0.004$ & $61.4 \pm 1.9$ & $5.10 \pm 0.19$ \\
\hline F50 & $198 \pm 3$ & $11.6 \pm 0.2$ & $0.768 \pm 0.006$ & $59.7 \pm 3.1$ & $5.32 \pm 0.24$ \\
\hline
\end{tabular}


the absorption and emission properties of the CT state are very similar in these three $50 \% \mathrm{~F}$ blends, irrespective of the means of fluorination (the EQE spectra of the HTAZ:PCBM and FTAZ:PCBM blends are displayed in Figure S13 in the SI).

The energy of the CT state $\left(E_{\mathrm{CT}}\right)$ was obtained by the combined fit of the $\mathrm{EQE}_{\mathrm{PV}}$ and $\mathrm{EQE}_{\mathrm{EL}}$ spectra with Gaussian line shape as outlined by Vandewal et al. ${ }^{49}$ The results of these fits, listed in Table 2, reveal very similar values for the CT

Table 2. Charge Transfer State Energy and Open-Circuit Voltage Losses as Denoted by $\Delta$

\begin{tabular}{|c|c|c|c|c|c|c|}
\hline & $\begin{array}{c}E_{\mathrm{CT}} \\
{[\mathrm{eV}]}\end{array}$ & $\begin{array}{c}V_{\mathrm{OC}, \text { rad }} \\
{[\mathrm{V}]}\end{array}$ & $\begin{array}{c}\Delta V_{\text {OC,rad. }} \\
{[\mathrm{V}]^{2}}\end{array}$ & $\begin{array}{c}\Delta V_{\mathrm{OC}, \text { nonrad. }} \\
{[\mathrm{V}]}\end{array}$ & $\begin{array}{c}V_{\text {OC,meas. }} \\
{[\mathrm{V}]}\end{array}$ & $\begin{array}{c}V_{\mathrm{OC}_{\text {, calc. }}} \\
{[\mathrm{V}]^{a}}\end{array}$ \\
\hline monoF & 1.38 & 1.14 & 0.24 & 0.366 & 0.761 & 0.774 \\
\hline $\mathrm{H}: \mathrm{F} \quad 1: 1$ & 1.38 & 1.15 & 0.23 & 0.373 & 0.764 & 0.777 \\
\hline F50 & 1.37 & 1.11 & 0.26 & 0.337 & 0.762 & 0.773 \\
\hline
\end{tabular}

energy for the three $50 \% \mathrm{~F}$ systems, at $1.37-1.38 \mathrm{eV}$, lying well between the CT energies of HTAZ $(1.34 \mathrm{eV})$ and FTAZ (1.40 $\mathrm{eV}$ ) (Figure S13). We also find very similar values for the Gaussian line width, suggesting the overall CT energetics to be independent of how fluorination was introduced into the blend. Note that the $E_{\mathrm{CT}}$ 's of the nonfluorinated, the $50 \%$ fluorinated, and the fully fluorinated blend differ only little, by a few tens of $\mathrm{meV}$, but that these differences are well discernible in the EQE spectra shown in Figure 3 and Figure S13. The very similar CT properties of the three $50 \% \mathrm{~F}$ systems, nonetheless being distinctly different from the properties of the nonfluorinated and fluorinated blends, indicate that they are mainly determined by the average concentration-rather than the exact local distribution - of the fluorinated units in the blend, in full agreement with recent simulation work which highlighted the importance of long-range electrostatic interactions in determining the CT energy. ${ }^{50,51}$

Comparison of $E_{\mathrm{CT}}$ and the measured $V_{\mathrm{oc}}$ also implies very similar values for the total $V_{\mathrm{oc}}$ loss, $E_{\mathrm{CT}}-\mathrm{e} V_{\mathrm{oc}}$ which is about $0.614 \pm 0.006 \mathrm{eV}$ in all $50 \% \mathrm{~F}$ systems. To quantify contributions by radiative and nonradiative recombination to this loss, the EQE spectra were analyzed according to

$$
\begin{gathered}
V_{\mathrm{OC}}=V_{\mathrm{OC}, \text { rad }}-\Delta V_{\mathrm{OC}, \text { nonrad }}=\frac{k_{\mathrm{B}} T}{q} \ln \frac{J_{\mathrm{G}}}{J_{0}} \\
=\frac{k_{\mathrm{B}} T}{q} \ln \frac{J_{\mathrm{Ph}}}{J_{0, \mathrm{Rad}}}+\frac{k_{\mathrm{B}} T}{q} \ln \left(\mathrm{EQE}_{\mathrm{EL}, \text { int }}\right)
\end{gathered}
$$

Here, $\mathrm{EQE}_{\mathrm{EL}, \text { int }}$ is the $\mathrm{EQE}_{\mathrm{EL}}$ integrated over the full spectrum; $q$ is the elementary charge; $h$ is Planck's constant; and $c$ denotes the speed of light. $V_{\mathrm{OC} \text {,rad }}$ is the open-circuit voltage in the case of purely radiative recombination $\left(\mathrm{EQE}_{\mathrm{EL} \text {,int }}=1\right)$, which is a sole function of the temperature, the generated photocurrent $\left(J_{\mathrm{Ph}}\right)$, and the radiative dark saturation current $\left(J_{0, \mathrm{Rad}}\right)$. Further, the radiative dark saturation current can be calculated from the photocurrent $\mathrm{EQE}_{\mathrm{PV}}$ spectra and the blackbody radiation $\left(\Phi_{\mathrm{BB}}\right)$ by $^{49}$

$$
J_{0, \mathrm{rad}}=q \int \mathrm{EQE}_{\mathrm{PV}}(\hbar \omega) \Phi_{\mathrm{BB}}(\hbar \omega) \mathrm{d} \hbar \omega
$$

To extend the directly measured $\mathrm{EQE}_{\mathrm{PV}}$ to lower photon energies (where $\Phi_{\mathrm{BB}}$ is largest), we make use of the optical reciprocity $\mathrm{EQE}_{\mathrm{PV}}(\hbar \omega) \propto \mathrm{EQE}_{\mathrm{EL}}(\hbar \omega) / \Phi_{\mathrm{BB}}(\hbar \omega) .^{52,53}$ In Figure 3, $\mathrm{EQE}_{\mathrm{PV}}$ derived from the $\mathrm{EQE}_{\mathrm{EL}}$ spectrum is depicted as a gray line. Its course matches very well that of the directly measured $\mathrm{EQE}_{\mathrm{PV}}$, indicating that the reciprocity between emission and absorption holds true for the chosen injection conditions (room-temperature conditions).

Having determined $\mathrm{EQE}_{\mathrm{PV}}(\hbar \omega)$ over a wide spectral range, the radiative limit of the open circuit voltage $\left(V_{\mathrm{OC}, \mathrm{rad}}\right)$ was calculated with eqs 2 and 3. The result is shown in Table 2 for all systems, together with values for the radiative $V_{\mathrm{OC}}$ loss $\left(\Delta V_{\mathrm{OC}, \text { rad }}=E_{\mathrm{CT}}-e V_{\mathrm{OC}, \text { rad }}\right)$, the nonradiative loss $\Delta V_{\mathrm{OC} \text {,nonrad, }}$ and $V_{\mathrm{OC}}$ calculated via $V_{\mathrm{OC}}=V_{\mathrm{OC}, \text { rad }}-\Delta V_{\mathrm{OC} \text {,nonrad. }}$ The agreement between the calculated $V_{\mathrm{OC}}$ with the measured $V_{\mathrm{OC}}$ (taken from the $J V$ measurement) is excellent, with a deviation less than $20 \mathrm{mV}$ for all systems. These results not only highlight the accuracy of the approach taken but also emphasize that the individual loss components are nearly the same in all three blends.

3.4. Free Charge Carrier Recombination. In order to more thoroughly understand the mechanism and kinetics of free charge carrier recombination, time-delayed collection field (TDCF) and bias-assisted charge extraction (BACE) were performed. These techniques have been used extensively and are described to a greater detail elsewhere. ${ }^{17,38,42,54-56}$ In TDCF, the sample is illuminated with a ns laser pulse while under a prebias $V_{\text {pre }}$. The bias is then switched to a large reverse bias $\left(V_{\text {coll }}\right)$ after a given delay time $t_{\mathrm{d}}$ to collect all free carriers which remain in the device at $t_{\mathrm{d}}$. TDCF thus allows us to investigate free charge generation and recombination upon pulsed illumination in the actual solar cell. BACE is a similar technique, but instead of a pulsed laser, a steady state illumination source is used. BACE measurements as a function of illumination intensity and prebias, therefore, reveal all parameters determining the steady state charge carrier density in the device, namely, the nongeminate recombination coefficient and the effective mobility of the photogenerated carriers. $^{56}$

3.4.1. Steady State Recombination. The charge carrier density at $V_{\mathrm{OC}}$ for different illumination conditions was measured via the BACE technique. At $V_{\mathrm{OC}}$, the recombination rate $R=-\gamma n^{\beta}$ is equal to the generation rate $G$ which itself is proportional to the light intensity. Here the recombination rate $R=-\gamma n^{\beta}$ is given in a very general way with $\beta$ as the recombination order and $\gamma$ as the recombination coefficient. By evaluating the extracted charge carrier density dependent on the illumination intensity, we are able to determine the recombination order. We find that $\beta$ is very close to 2 (Figure S14) for all three systems, indicating that the main recombination channel is bimolecular recombination (BMR).

3.4.2. Transient Nongeminate Recombination. Transient recombination experiments were conducted using the TDCF technique as outlined above. These studies were motivated by our recent report of pronounced dispersive recombination in the binary blend of the donor polymer PCDTBT with $\mathrm{PC}_{61} \mathrm{BM}^{57}$ It was concluded that energetic disorder results in a long-term thermalization of carriers, resulting in a slow down of carrier motion and with that slowing of the rate of nongeminate recombination. In our transient recombination experiments, extraction after excitation with a laser pulse was varied between approximately $5 \mathrm{~ns}$ to a few $\mu$ s. From the totally extracted charge density $n_{\text {tot }}$ as a function of delay time $t_{\mathrm{d}}$, the recombination rate $R$ is calculated via $R\left(t_{\mathrm{d}}\right)=\frac{\Delta n_{\text {tot }}\left(t_{\mathrm{d}}\right)}{\Delta t_{\mathrm{d}}}$. From these data, differential decay plots are generated by plotting $R$ as a function of the collected charge $n_{\text {coll }}\left(t_{\mathrm{d}}\right)$ (the carrier density 
present in the device at time $t_{\mathrm{d}}$ ). Figure 4 summarized such differential decay plots for all three $50 \% \mathrm{~F}$ systems for a prebias of $0.76 \mathrm{~V}$ (open-circuit conditions).

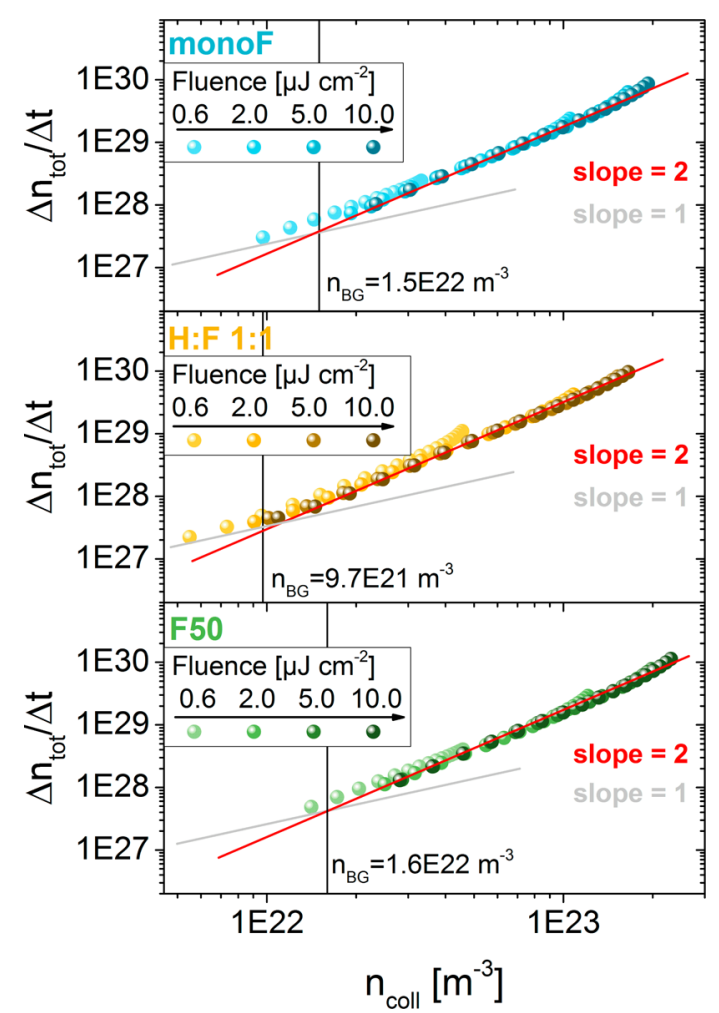

Figure 4. Differential decay of the total charge carrier density extracted in the TDCF experiment plotted versus the collected charge carrier density. The decay was measured for a broad intensity range as denoted in the legend. The concentration of background charge for each system is shown as a vertical black line. The red and gray lines with a slope of 2 and 1, respectively, are guides to the eye to show the apparent recombination order. As predicted by eq 4 , the order is one at low $n_{\text {coll }}$ (recombination of photogenerated charge with dark charge) and increases to two at high $n_{\text {coll }}$ (when recombination is predominately between photogenerated charge).

In general, these plots can be subdivided into two regions. ${ }^{55,57}$ At short delay times $t_{\mathrm{d}}$, the recombination rate depends explicitly on $t_{\mathrm{d}}$. This is the regime of dispersive recombination, where the recombination dynamics are affected by the slow-down of charge carrier motion due to thermalization. At longer times, the differential decay data for different fluences fall into one line, meaning that the recombination rate becomes an explicit function of only the carrier density (but not of the delay time). This is the regime governed by the recombination of thermalized charges.

Importantly, the differential decays of the three $50 \% \mathrm{~F}$ systems display significant similarities in every respect. First, in all three systems, there is only minor (if not negligible) contribution from dispersive recombination. Given that the time scale and extent of carrier thermalization and recombination depend largely on the type of energetic disorder, ${ }^{58}$ our finding suggests that the relevant density of state distributions of the three blends are very similar or that any difference is not relevant for the recombination dynamics at the measured time scale. Second, in the regime of thermalized carrier recombination, not only the overall power-law dependence of $R$ or $n_{\text {coll }}$ but also the absolute recombination rate for a given carrier density are very similar for the three blends. In fact, by additionally accounting for injected dark charges $\left(n_{\mathrm{BG}}\right)$, which are determined by $\mathrm{BACE},{ }^{59}$ the differential decay of the total charge carrier density can be fitted by

$$
\frac{\Delta n_{\mathrm{tot}}}{\Delta t}=-k_{2}\left(n_{\mathrm{coll}}^{2}+2 n_{\mathrm{coll}} n_{\mathrm{BG}}\right)
$$

The resulting BMR coefficients $k_{2}$, obtained via fitting eq 4 , are comparable to the coefficients from BACE and are found to be $1.2 \times 10^{-17} \mathrm{~m}^{3} \mathrm{~s}^{-1}$ for F50, $1.0 \times 10^{-17} \mathrm{~m}^{3} \mathrm{~s}^{-1}$ for monoF and 2 $\times 10^{-17} \mathrm{~m}^{3} \mathrm{~s}^{-1}$ for $\mathrm{H}: \mathrm{F} 1: 1$.

Overall, TDCF data also show very comparable results for all three systems, indicating that the recombination mechanism and pathway are largely identical, regardless of how the fluorine substitution is incorporated into the bulk heterojunction. This finding is in especially good agreement with the similar voltage loss (vide supra), indicating that the mesoscale morphology determines the CT-state energetics and, with that, also the bimolecular recombination mechanism.

3.5. Electron and Hole Mobility. Single carrier mobilities were measured using the space-charge limited current (SCLC) method. The results are included in Table 3. For the 50\% blends (monoF, F50, and $\mathrm{H}: \mathrm{F} 1: 1$ ), the hole mobilities are similar and range from 3 to $6.7 \times 10^{-4} \mathrm{~cm}^{2} \mathrm{~V}^{-1} \mathrm{~s}^{-1}$. These values lie between the mobilities of HTAZ and FTAZ and agree with the similar FF for the $50 \%$ blends. The electron mobilities for the 50\% devices are all similar at $1-2 \times 10^{-3} \mathrm{~cm}^{2} \mathrm{~V}^{-1} \mathrm{~s}^{-1}$ and agree well with the previous measurements. ${ }^{17}$ As those values have been obtained on single carrier devices, we also measured the effective steady state mobility in the actual solar cell with BACE, following the procedure published by Albrecht et al. ${ }^{56}$ Specifically, we measured the extracted charges at different open-circuit voltages (for different illumination intensity) and at different voltages for fixed illumination intensities, ${ }^{56}$ with data collected in Figure $S 15$ in the Supporting Information. The resulting effective mobilities are also listed in Table 3. These values are then compared to effective mobility calculated from the single carrier mobilities via

$$
\mu_{\text {eff,calc }}=\frac{2 \mu_{\mathrm{e}} \mu_{\mathrm{h}}}{\mu_{\mathrm{e}}+\mu_{\mathrm{h}}}
$$

Table 3. Hole and Electron Mobilities as Measured Using SCLC

$\begin{array}{lcccc} & \mu_{\mathrm{h}}\left[10^{-4} \mathrm{~cm}^{2} \mathrm{~V}^{-1} \mathrm{~s}^{-1}\right] & \mu_{\mathrm{e}}\left[10^{-4} \mathrm{~cm}^{2} \mathrm{~V}^{-1} \mathrm{~s}^{-1}\right] & \mu_{\text {eff,calc. }}\left[10^{-4} \mathrm{~cm}^{2} \mathrm{~V}^{-1} \mathrm{~s}^{-1}\right] & \mu_{\text {eff,meas }}\left[10^{-4} \mathrm{~cm}^{2} \mathrm{~V}^{-1} \mathrm{~s}^{-1}\right] \\ \text { HTAZ } & 2 \pm 0.4 & -- & -- & - \\ \text { FTAZ } & 22.2 \pm 5.6 & - & - & - \\ \text { monoF } & 4.4 \pm 0.4 & 12 \pm 5 & 6.4 & 5.3 \pm 0.7 \\ \text { H:F 1:1 } & 6.7 \pm 1.7 & 18 \pm 8 & 9.7 & 7 \pm 2 \\ \text { F50 } & 3.0 \pm 0.3 & 23 \pm 7 & 5.3 & 5.5 \pm 0.1\end{array}$


We have observed good agreement between the calculated $\mu_{\text {eff }}$ and the measured $\mu_{\text {eff }}$ indicating that the electron and hole mobilities of the unipolar devices are also relevant for the performance of the bipolar solar cell device. Therefore, we can safely conclude that the extraction of photogenerated charge in the solar cell device is limited by the lower mobility -in this study, the hole mobility, which is very similar for all three $50 \%$ F systems.

3.6. Morphology of Three $50 \%$ F Blends. Transmission electron microscopy (TEM) was used to investigate the morphology of these 50\% F-based $\mathrm{BHJ}$ blends. Specifically, the polymer: $\mathrm{PC}_{61} \mathrm{BM} \mathrm{BHJ}$ blends were cast on $\mathrm{Si}$ substrates coated with unannealed PEDOT:PSS using the same casting parameters used to fabricate the same $\mathrm{BHJ}$ blend based devices, yielding films of $\sim 200 \mathrm{~nm}$ thickness. These $\mathrm{BHJ}$ blends were then floated onto TEM grids for measurement by dissolving the unannealed PEDOT:PSS sacrificial layer. Both bright-field images (zero loss) and sulfur maps generated from energyfiltered TEM micrographs were acquired and are presented in Figure 5 .

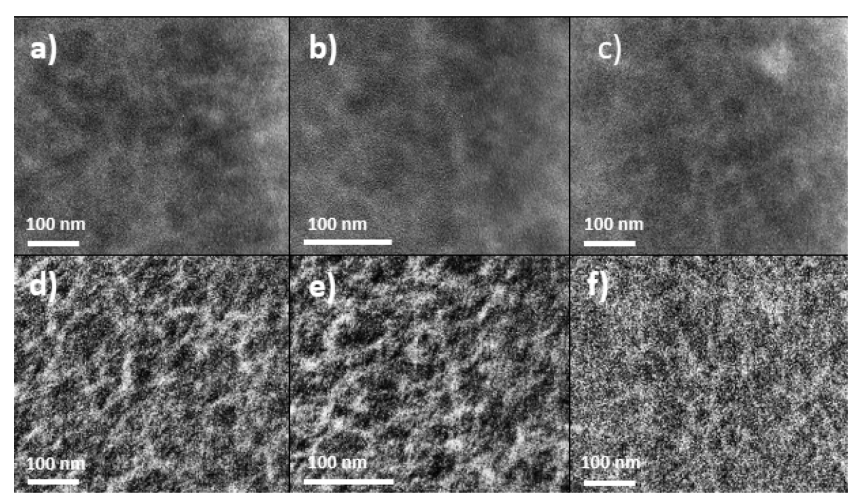

Figure 5. TEM images of the thick $(\sim 200 \mathrm{~nm})$, device relevant films. Bright field (zero-loss) of (a) H:F 1:1, (b) monoF, and (c) F50 and sulfur maps of (d) H:F 1:1, (e) monoF, and (f) F50 generated from the standard three-window method.

Overall, the three 50\% F-based BHJ blends show morphology characteristic of most $\mathrm{BHJ}$ polymer solar cells. There are no significant differences in morphology discernible from these images. ${ }^{60}$ However, the rather large thickness of the device relevant films $(\sim 200 \mathrm{~nm})$ may contribute to a lower quality image since TEM is a transmission based technique. Therefore, thinner films $(\sim 100 \mathrm{~nm})$ were also fabricated and characterized by TEM (Figure S16). Indeed, these thinner films were able to offer improved image quality but still show a similar morphology for all three 50\% F-based $\mathrm{BHJ}$ blends, verifying the conclusions reached from the device relevant thicknesses.

The similar morphology for all three 50\% F-based $\mathrm{BHJ}$ blends, as seen from the TEM images, is especially interesting since one might expect increased phase separation from the $\mathrm{H}: \mathrm{F} 1: 1$ blend due to the ternary nature of the blend. However, as with these two binary BHJ blends (i.e., F50 and monoF), no coarse, unfavorable phase separation is apparent in the films of the H:F 1:1 based ternary blend (Figure 5). Given this observation, and our earlier discovery of similar morphology features between HTAZ and FTAZ based binary blends (e.g., similar domain purity and miscibility with $\left.\mathrm{PC}_{61} \mathrm{BM}\right),{ }^{11,17}$ we suggest that the miscibility of HTAZ and FTAZ is very high and plays a key role in their cooperative behavior. Indeed, if
HTAZ and FTAZ were largely "miscible", then these three 50\% $\mathrm{F}$ systems would be essentially identical HTAZ:PC ${ }_{61} \mathrm{BM}$ binary blends with the same amount of $\mathrm{F}$ atom incorporated (i.e., $50 \%$ F)! This observation would explain the similar device performance and device physics, as well as the similar morphology for these three $50 \% \mathrm{~F}$ systems.

3.7. Performance of HTAZ:FTAZ Physical Blends. If the physical blending of HTAZ and FTAZ was able to offer similar device performance to that of the corresponding random copolymer at similar fluorination level, as we have seen in the case of $50 \% \mathrm{~F}$, then such physical blends at different ratios (e.g., $25 \%)$ would also perform similarly to their random copolymer counterparts. We therefore expanded our study to consider the physical blends of HTAZ and FTAZ at 1:3 and 3:1 ratios.

The device performance of the physical blends at ratios of $\mathrm{H}: \mathrm{F}$ 3:1 and H:F 1:3, together with 1:1, HTAZ and FTAZ, is included in the Supporting Information (Table S3). Indeed, the device performance of the physical blends follows the same trend as the previously published H/FTAZ random copolymers: improved performance with increased fluorine. ${ }^{17}$ Specifically, the $J_{\mathrm{sc}}$ of the 1:3,1:1, and 3:1 blends lies between those of HTAZ and FTAZ, but this minute variation of $J_{\text {sc }}$ cannot account for the continual increase in performance as the percentage of FTAZ in the physical blend increases. The $V_{\mathrm{oc}}$ also increases very slowly from $0.76 \mathrm{~V}$ from $0 \%$ FTAZ (i.e., HTAZ) to $0.81 \mathrm{~V}$ with $100 \%$ FTAZ, similar to what we observed for the H/FTAZ random copolymers. Most interestingly, the FF makes a marked improvement with increased percentage of FTAZ, which closely follows the overall improvement in PCE. This observation strikingly mirrors what was discovered in the previously investigated H/FTAZ random copolymers, where the FF was the main contributor to increased performance with increased fluorination. It is intriguing that we observe the same trend when physically mixing a fluorinated polymer (FTAZ) and a nonfluorinated polymer (HTAZ), rather than directly fluorinating the backbone. This observation also provides further evidence to suggest that HTAZ and FTAZ are "miscible."

In our previous study on the H/FTAZ-based random copolymers, the monotonically increasing hole mobility with fluorination was identified as the sole cause to account for the continuously improved FF (and PCE). We then measured the hole mobilities of these physical blends via the SCLC method and observed a similar behavior: the mobilities track well with the FF and the PCE, increasing as the percentage of FTAZ in the physical blend is increased (Figure 6). This observation demonstrates that it is possible to use a high mobility polymer (e.g., FTAZ) to improve the overall mobility of a ternary blend (e.g., HTAZ:FTAZ). More importantly, our discovery might offer an alternative strategy to improve the overall efficiency of polymer solar cells by enhancing the hole mobility via a physical blend. Many small band gap polymers suffer from relatively low mobility. For example, the high performing PTB7 has a relatively low mobility of $5.8 \times 10^{-4} \mathrm{~cm}^{2} \mathrm{~V}^{-1} \mathrm{~s}^{-1}$, limiting the active layer thickness to $\sim 100 \mathrm{~nm} .^{6}$ Such a thin layer seriously restricts the light absorption and the $J_{s c}$ and also imposes technical difficulty on future roll-to-roll printed solar cells. We thus propose that it is possible to both improve the charge transport (i.e., high mobility) and extend the light absorption (i.e., a small band gap) by incorporating a medium to high band gap, high mobility polymer (such as FTAZ) into a ternary blend system with a low mobility, small band gap polymer. This, of course, assumes that the components form a 


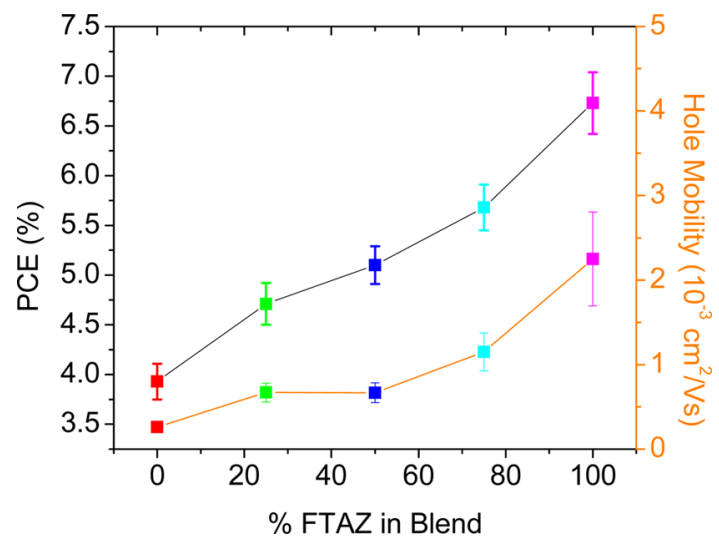

Figure 6. Hole mobility and power conversion efficiency graphed vs the amount of fluorinated polymer in the physical blends.

favorable morphology. As previously discussed, it is likely that HTAZ and FTAZ are miscible and form the favorable morphology necessary for beneficial cooperation.

\section{CONCLUSION}

In summary, we have compared three systems with equal amounts of fluorination (50\%) achieved through different means. It is shown that all three systems-monoF, F50, and $\mathrm{H}: \mathrm{F}$ 1:1 physical blend-have similarly improved device performance over the nonfluorinated system in every respect. Generation of free charges is shown to be field independent in all three systems, which implies that the curvature of the $J-V$ is determined by the extraction and recombination of free charges. Transient (TDCF) and steady state (BACE) measurements prove that the recombination is bimolecular, and the recombination coefficients vary only a little between the systems. The similarity of the recombination mechanism in all systems is in agreement with the results from the analysis of the photovoltaic and electroluminescent external quantum efficiency spectra, which yielded very little differences of the CT properties among the three $50 \% \mathrm{~F}$ systems (i.e., random, regular, and physical blend). This important finding supports the picture that CT properties of organic donor-acceptor blends are indeed affected by longer range electrostatic interactions and not simply determined by the properties of individual donor-acceptor pairs at the donor-acceptor interface. ${ }^{50,51}$ Effective mobilities were determined with two independent methods (SCLC and BACE) and found to be very similar for the three systems. The almost identical values for the generation efficiency, the effective mobility, and the bimolecular recombination coefficient explain why the fill factor (FF) varies so little among all three systems.

The improved performance over the nonfluorinated systems stems largely from an improved hole mobility, leading to a higher fill factor of these polymer-based devices. This improvement is especially noteworthy for the physical blend, where the likely miscibility of the two polymers allows for the improved performance. We believe that this mobility enhancement via a physical blend could be employed to improve a low mobility system with the addition of a high mobility component. Importantly, if these polymers also have complementary absorbance and similar $V_{o c}$ increased $J_{s c}$ and high $V_{\text {oc }}$ may be obtained concurrently with the improved FF. This new approach would disentangle the synthetic restraints on the donor material and open alternative routes to high performing polymer solar cells.

\section{ASSOCIATED CONTENT}

Supporting Information

The Supporting Information is available free of charge on the ACS Publications website at DOI: 10.1021/acs.jpcc.6b10993.

Synthetic details, scheme, NMR, GPC curves, UV-vis spectra, and elemental analysis of monoFTAZ. CV scans and HOMO energy levels for all polymers. UV-vis, EQE, and EGE of the $50 \%$ blends. Extracted carrier densities. TEM of thin films. PV Performance of the physical blends (PDF)

\section{AUTHOR INFORMATION}

\section{Corresponding Authors}

*E-mail: neher@uni-potsdam.de.

*E-mail: wyou@unc.edu.

ORCID ${ }^{\circ}$

Wei You: 0000-0003-0354-1948

\section{Author Contributions}

${ }^{\#}$ M.A.K. and S.R. contributed equally. The manuscript was written through contributions of all authors. All authors have given approval to the final version of the manuscript.

\section{Notes}

The authors declare no competing financial interest.

\section{ACKNOWLEDGMENTS}

MAK, QZ, and WY were supported by a NSF grant (DMR1507249) and the Office of Naval Research (Grant No. N000141410221). DN and SR acknowledge financial support by the German Ministry of Science and Education (Project UNVEIL, FKZ 13N13719). YL, BK, QW, and EDG acknowledge financial support from the Office of Naval Research, United States, under Grant no. N000141410532

\section{REFERENCES}

(1) Price, S. C.; Stuart, A. C.; Yang, L.; Zhou, H.; You, W. Fluorine Substituted Conjugated Polymer of Medium Band Gap Yields 7\% Efficiency in Polymer-Fullerene Solar Cells. J. Am. Chem. Soc. 2011, 133, 4625-4631.

(2) Leclerc, N.; Chávez, P.; Ibraikulov, O.; Heiser, T.; Lévêque, P. Impact of Backbone Fluorination on $\pi$-Conjugated Polymers in Organic Photovoltaic Devices: A Review. Polymers (Basel, Switz.) 2016, 8, 11.

(3) Zhou, H.; Yang, L.; Stuart, A. C.; Price, S. C.; Liu, S.; You, W. Development of Fluorinated Benzothiadiazole as a Structural Unit for a Polymer Solar Cell of 7\% Efficiency. Angew. Chem., Int. Ed. 2011, 50, 2995-2998.

(4) Uy, R. L.; Price, S. C.; You, W. Structure-Property Optimizations in Donor Polymers via Electronics, Substituents, and Side Chains Toward High Efficiency Solar Cells. Macromol. Rapid Commun. 2012, 33, 1162-1177.

(5) Zhou, H.; Yang, L.; You, W. Rational Design of High Performance Conjugated Polymers for Organic Solar Cells. Macromolecules 2012, 45, 607-632.

(6) Liang, Y.; Xu, Z.; Xia, J.; Tsai, S.-T.; Wu, Y.; Li, G.; Ray, C.; Yu, L. For the Bright Future-Bulk Heterojunction Polymer Solar Cells with Power Conversion Efficiency of 7.4\%. Adv. Mater. 2010, 22, E135E138.

(7) Wan, Q.; Guo, X.; Wang, Z.; Li, W.; Guo, B.; Ma, W.; Zhang, M.; Li, Y. 10.8\% Efficiency Polymer Solar Cells Based on PTB7-Th and PC 71 BM via Binary Solvent Additives Treatment. Adv. Funct. Mater. 2016, 26, 6635-6640. 
(8) Zhou, H.; Yang, L.; Stuart, A. C.; Price, S. C.; Liu, S.; You, W. Development of Fluorinated Benzothiadiazole as a Structural Unit for a Polymer Solar Cell of 7\% Efficiency. Angew. Chem., Int. Ed. 2011, 50, 2995-2998.

(9) Chen, C.-P.; Chen, Y.-C.; Yu, C.-Y. Increased Open Circuit Voltage in a Fluorinated Quinoxaline-Based Alternating Conjugated Polymer. Polym. Chem. 2013, 4, 1161-1166.

(10) Cartwright, L.; Iraqi, A.; Zhang, Y.; Wang, T.; Lidzey, D. G. Impact of Fluorine Substitution upon the Photovoltaic Properties of Benzothiadiazole-Fluorene Alternate Copolymers. RSC Adv. 2015, 5, 46386-46394.

(11) Stuart, A. C.; Tumbleston, J. R.; Zhou, H.; Li, W.; Liu, S.; Ade, H.; You, W. Fluorine Substituents Reduce Charge Recombination and Drive Structure and Morphology Development in Polymer Solar Cells. J. Am. Chem. Soc. 2013, 135 (5), 1806-1815.

(12) Son, H. J.; Wang, W.; Xu, T.; Liang, Y.; Wu, Y.; Li, G.; Yu, L. Synthesis of Fluorinated Polythienothiophene-Co-Benzodithiophenes and Effect of Fluorination on the Photovoltaic Properties. J. Am. Chem. Soc. 2011, 133, 1885-1894.

(13) Fei, Z.; Boufflet, P.; Wood, S.; Wade, J.; Moriarty, J.; Gann, E.; Ratcliff, E. L.; McNeill, C. R.; Sirringhaus, H.; Kim, J.-S.; et al. Influence of Backbone Fluorination in Regioregular Poly(3-Alkyl-4Fluoro)thiophenes. J. Am. Chem. Soc. 2015, 137, 6866-6879.

(14) Zhang, Y.; Chien, S.-C.; Chen, K.-S.; Yip, H.-L.; Sun, Y.; Davies, J. A.; Chen, F.-C.; Jen, A. K.-Y. Increased Open Circuit Voltage in Fluorinated Benzothiadiazole-Based Alternating Conjugated Polymers. Chem. Commun. 2011, 47, 11026.

(15) Son, H. J.; Wang, W.; Xu, T.; Liang, Y.; Wu, Y.; Li, G.; Yu, L. Synthesis of Fluorinated Polythienothiophene- Co -Benzodithiophenes and Effect of Fluorination on the Photovoltaic Properties. J. Am. Chem. Soc. 2011, 133, 1885-1894.

(16) Liang, Y.; Feng, D.; Wu, Y.; Tsai, S.-T.; Li, G.; Ray, C.; Yu, L. Highly Efficient Solar Cell Polymers Developed via Fine-Tuning of Structural and Electronic Properties. J. Am. Chem. Soc. 2009, 131, 7792-7799.

(17) Li, W.; Albrecht, S.; Yang, L.; Roland, S.; Tumbleston, J. R.; McAfee, T.; Yan, L.; Kelly, M. A.; Ade, H.; Neher, D.; et al. MobilityControlled Performance of Thick Solar Cells Based on Fluorinated Copolymers. J. Am. Chem. Soc. 2014, 136, 15566-15576.

(18) Nketia-Yawson, B.; Lee, H.-S.; Seo, D.; Yoon, Y.; Park, W.-T.; Kwak, K.; Son, H. J.; Kim, B.; Noh, Y.-Y. A Highly Planar Fluorinated Benzothiadiazole-Based Conjugated Polymer for High-Performance Organic Thin-Film Transistors. Adv. Mater. 2015, 27, 3045-3052.

(19) Jung, J. W.; Liu, F.; Russell, T. P.; Jo, W. H. Medium Bandgap Conjugated Polymer for High Performance Polymer Solar Cells Exceeding 9\% Power Conversion Efficiency. Adv. Mater. 2015, 27, $7462-7468$.

(20) Jo, J. W.; Jung, J. W.; Wang, H.-W.; Kim, P.; Russell, T. P.; Jo, W. H. Fluorination of Polythiophene Derivatives for High Performance Organic Photovoltaics. Chem. Mater. 2014, 26, 4214-4220.

(21) Fei, Z.; Shahid, M.; Yaacobi-Gross, N.; Rossbauer, S.; Zhong, H.; Watkins, S. E.; Anthopoulos, T. D.; Heeney, M. Thiophene Fluorination to Enhance Photovoltaic Performance in Low Band Gap Donor-acceptor Polymers. Chem. Commun. 2012, 48, 11130.

(22) Tumbleston, J. R.; Stuart, A. C.; Gann, E.; You, W.; Ade, H. Fluorinated Polymer Yields High Organic Solar Cell Performance for a Wide Range of Morphologies. Adv. Funct. Mater. 2013, 23, 34633470.

(23) Liu, Y.; Zhao, J.; Li, Z.; Mu, C.; Ma, W.; Hu, H.; Jiang, K.; Lin, H.; Ade, H.; Yan, H. Aggregation and Morphology Control Enables Multiple Cases of High-Efficiency Polymer Solar Cells. Nat. Commun. 2014, 5, 5293.

(24) Zhao, J.; Li, Y.; Yang, G.; Jiang, K.; Lin, H.; Ade, H.; Ma, W.; Yan, H. Efficient Organic Solar Cells Processed from Hydrocarbon Solvents. Nat. Energy 2016, 1, 15027.

(25) Li, Z.; Lin, H.; Jiang, K.; Carpenter, J.; Li, Y.; Liu, Y.; Hu, H.; Zhao, J.; Ma, W.; Ade, H.; et al. Dramatic Performance Enhancement for Large Bandgap Thick-Film Polymer Solar Cells Introduced by a Difluorinated Donor Unit. Nano Energy 2015, 15, 607-615.
(26) Zhang, Q.; Kelly, M. A.; Hunt, A.; Ade, H.; You, W. Comparative Photovoltaic Study of Physical Blending of Two Donor-Acceptor Polymers with the Chemical Blending of the Respective Moieties. Macromolecules 2016, 49, 2533-2540.

(27) Yang, L.; Zhou, H.; Price, S. C.; You, W. Parallel-like Bulk Heterojunction Polymer Solar Cells. J. Am. Chem. Soc. 2012, 134, $5432-5435$

(28) Yang, L.; Yan, L.; You, W. Organic Solar Cells beyond One Pair of Donor-Acceptor: Ternary Blends and More. J. Phys. Chem. Lett. 2013, 4, 1802-1810.

(29) Lu, L.; Chen, W.; Xu, T.; Yu, L. High-Performance Ternary Blend Polymer Solar Cells Involving Both Energy Transfer and Hole Relay Processes. Nat. Commun. 2015, 6, 7327.

(30) Ameri, T.; Khoram, P.; Min, J.; Brabec, C. J. Organic Ternary Solar Cells: A Review. Adv. Mater. 2013, 25, 4245-4266.

(31) Mollinger, S. A.; Vandewal, K.; Salleo, A. Microstructural and Electronic Origins of Open-Circuit Voltage Tuning in Organic Solar Cells Based on Ternary Blends. Adv. Energy Mater. 2015, 5, 1501335.

(32) Street, R. a; Davies, D.; Khlyabich, P. P.; Burkhart, B.; Thompson, B. C. Origin of the Tunable Open-Circuit Voltage in Ternary Blend Bulk Heterojunction Organic Solar Cells. J. Am. Chem. Soc. 2013, 135, 986-989.

(33) Felekidis, N.; Wang, E.; Kemerink, M. Open Circuit Voltage and Efficiency in Ternary Organic Photovoltaic Blends. Energy Environ. Sci. 2016, 9, 257-266.

(34) Tietze, M. L.; Tress, W.; Pfützner, S.; Schünemann, C.; Burtone, L.; Riede, M.; Leo, K.; Vandewal, K.; Olthof, S.; Schulz, P.; et al. Correlation of Open-Circuit Voltage and Energy Levels in ZincPhthalocyanine: C 60 Bulk Heterojunction Solar Cells with Varied Mixing Ratio. Phys. Rev. B: Condens. Matter Mater. Phys. 2013, 88, 85119.

(35) Kozub, D. R.; Vakhshouri, K.; Orme, L. M.; Wang, C.; Hexemer, A.; Gomez, E. D. Polymer Crystallization of Partially Miscible Polythiophene/Fullerene Mixtures Controls Morphology. Macromolecules 2011, 44, 5722-5726.

(36) Li, W.; Yang, L.; Tumbleston, J. R.; Yan, L.; Ade, H.; You, W. Controlling Molecular Weight of a High Efficiency Donor-Acceptor Conjugated Polymer and Understanding Its Significant Impact on Photovoltaic Properties. Adv. Mater. 2014, 26, 4456-4462.

(37) Cardona, C. M.; Li, W.; Kaifer, A. E.; Stockdale, D.; Bazan, G. C. Electrochemical Considerations for Determining Absolute Frontier Orbital Energy Levels of Conjugated Polymers for Solar Cell Applications. Adv. Mater. 2011, 23, 2367-2371.

(38) Roland, S.; Schubert, M.; Collins, B. A.; Kurpiers, J.; Chen, Z.; Facchetti, A.; Ade, H.; Neher, D. Fullerene-Free Polymer Solar Cells with Highly Reduced Bimolecular Recombination and FieldIndependent Charge Carrier Generation. J. Phys. Chem. Lett. 2014, $5,2815-2822$.

(39) Kniepert, J.; Schubert, M.; Blakesley, J. C.; Neher, D. Photogeneration and Recombination in P3HT/PCBM Solar Cells Probed by Time-Delayed Collection Field Experiments. J. Phys. Chem. Lett. 2011, 2, 700-705.

(40) Albrecht, S.; Schindler, W.; Kurpiers, J.; Kniepert, J.; Blakesley, J. C.; Dumsch, I.; Allard, S.; Fostiropoulos, K.; Scherf, U.; Neher, D. On the Field Dependence of Free Charge Carrier Generation and Recombination in Blends of PCPDTBT/PC70BM: Influence of Solvent Additives. J. Phys. Chem. Lett. 2012, 3, 640-645.

(41) Kniepert, J.; Lange, I.; Heidbrink, J.; Kurpiers, J.; Brenner, T. J. K.; Koster, L. J. A.; Neher, D. Effect of Solvent Additive on Generation, Recombination, and Extraction in PTB7:PCBM Solar Cells: A Conclusive Experimental and Numerical Simulation Study. J. Phys. Chem. C 2015, 119, 8310-8320.

(42) Kurpiers, J.; Balazs, D. M.; Paulke, A.; Albrecht, S.; Lange, I.; Protesescu, L.; Kovalenko, M. V; Loi, M. A.; Neher, D. Free Carrier Generation and Recombination in PbS Quantum Dot Solar Cells. submitted 2016

(43) Few, S.; Frost, J. M.; Nelson, J. Models of Charge Pair Generation in Organic Solar Cells. Phys. Chem. Chem. Phys. 2015, 17, 2311-2325. 
(44) Albrecht, S.; Janietz, S.; Schindler, W.; Frisch, J.; Kurpiers, J.; Kniepert, J.; Inal, S.; Pingel, P.; Fostiropoulos, K.; Koch, N.; et al. Fluorinated Copolymer PCPDTBT with Enhanced Open-Circuit Voltage and Reduced Recombination for Highly Efficient Polymer Solar Cells. J. Am. Chem. Soc. 2012, 134, 14932-14944.

(45) Zusan, A.; Vandewal, K.; Allendorf, B.; Hansen, N. H.; Pflaum, J.; Salleo, A.; Dyakonov, V.; Deibel, C. The Crucial Influence of Fullerene Phases on Photogeneration in Organic Bulk Heterojunction Solar Cells. Adv. Energy Mater. 2014, 4, 1400922.

(46) Gélinas, S.; Rao, A.; Kumar, A.; Smith, S. L.; Chin, A. W.; Clark, J.; van der Poll, T. S.; Bazan, G. C.; Friend, R. H. Ultrafast Long-Range Charge Separation in Organic Semiconductor Photovoltaic Diodes. Science 2014, 343, 512.

(47) Schwarz, C.; Tscheuschner, S.; Frisch, J.; Winkler, S.; Koch, N.; Bässler, H.; Köhler, A. Role of the Effective Mass and Interfacial Dipoles on Exciton Dissociation in Organic Donor-Acceptor Solar Cells. Phys. Rev. B: Condens. Matter Mater. Phys. 2013, 87, 155205.

(48) Stolterfoht, M.; Armin, A.; Philippa, B.; White, R. D.; Burn, P. L.; Meredith, P.; Juška, G.; Pivrikas, A. Photocarrier Drift Distance in Organic Solar Cells and Photodetectors. Sci. Rep. 2015, 5, 9949.

(49) Vandewal, K.; Tvingstedt, K.; Gadisa, A.; Inganäs, O.; Manca, J. V. Relating the Open-Circuit Voltage to Interface Molecular Properties of Donor:acceptor Bulk Heterojunction Solar Cells. Phys. Rev. B: Condens. Matter Mater. Phys. 2010, 81, 125204.

(50) Poelking, C.; Tietze, M.; Elschner, C.; Olthof, S.; Hertel, D.; Baumeier, B.; Würthner, F.; Meerholz, K.; Leo, K.; Andrienko, D. Impact of Mesoscale Order on Open-Circuit Voltage in Organic Solar Cells. Nat. Mater. 2014, 14, 434-439.

(51) Sweetnam, S.; Prasanna, R.; Burke, T. M.; Bartelt, J. A.; McGehee, M. D. How the Energetic Landscape in the Mixed Phase of Organic Bulk Heterojunction Solar Cells Evolves with Fullerene Content. J. Phys. Chem. C 2016, 120, 6427-6434.

(52) Rau, U. Reciprocity Relation between Photovoltaic Quantum Efficiency and Electroluminescent Emission of Solar Cells. Phys. Rev. B: Condens. Matter Mater. Phys. 2007, 76, 85303.

(53) Vandewal, K.; Albrecht, S.; Hoke, E. T.; Graham, K. R.; Widmer, J.; Douglas, J. D.; Schubert, M.; Mateker, W. R.; Bloking, J. T.; Burkhard, G. F.; et al. Efficient Charge Generation by Relaxed ChargeTransfer States at Organic Interfaces. Nat. Mater. 2013, 13, 63-68.

(54) Kniepert, J.; Schubert, M.; Blakesley, J. C.; Neher, D. Photogeneration and Recombination in P3HTPCBM Solar Cells Probed by Time-Delayed Collection Field Experiments. J. Phys. Chem. Lett. 2011, 2, 700-705.

(55) Paulke, A.; Stranks, S. D.; Kniepert, J.; Kurpiers, J.; Wolff, C. M.; Schön, N.; Snaith, H. J.; Brenner, T. J. K.; Neher, D. Charge Carrier Recombination Dynamics in Perovskite and Polymer Solar Cells. Appl. Phys. Lett. 2016, 108, 113505.

(56) Albrecht, S.; Tumbleston, J. R.; Janietz, S.; Dumsch, I.; Allard, S.; Scherf, U.; Ade, H.; Neher, D. Quantifying Charge Extraction in Organic Solar Cells: The Case of Fluorinated PCPDTBT. J. Phys. Chem. Lett. 2014, 5, 1131-1138.

(57) Kurpiers, J.; Neher, D. Dispersive Non-Geminate Recombination in an Amorphous Polymer:Fullerene Blend. Sci. Rep. 2016, 6, 26832.

(58) Bässler, H. Charge Transport in Disordered Organic Photoconductors a Monte Carlo Simulation Study. Phys. Status Solidi B 1993, 175, 15-56.

(59) Kniepert, J.; Lange, I.; Van Der Kaap, N. J.; Koster, L. J. A.; Neher, D. A Conclusive View on Charge Generation, Recombination, and Extraction in as-Prepared and Annealed P3HT:PCBM Blends: Combined Experimental and Simulation Work. Adv. Energy Mater. 2014, 4, 1301401.

(60) Kuei, B.; Gomez, E. D. Chain Conformations and Phase Behavior of Conjugated Polymers. Soft Matter 2016, 114, 1008710088. 